\title{
Emergence and Communication in Computational Sociology
}

\author{
Mauricio Salgado and Nigel Gilbert
}

\begin{abstract}
.
Computational sociology models social phenomena using the concepts of emergence and downward causation. However, the theoretical status of these concepts is ambiguous; they suppose too much ontology and are invoked by two opposed sociological interpretations of social reality: the individualistic and the holistic. This paper aims to clarify those concepts and argue in favour of their heuristic value for social simulation. It does so by proposing a link between the concept of emergence and Luhmann's theory of communication. For Luhmann, society emerges from the bottom-up as communication and he describes the process by which society limits the possible selections of individuals as downward causation. It is argued that this theory is well positioned to overcome some epistemological drawbacks in computational sociology.
\end{abstract}

Keywords: Communication, Computational Sociology, Downward Causation, Emergence, Niklas Luhmann.

The system emerges etsi non daretur Deus.

Niklas Luhmann (1996, p.105)

\section{Introduction}

Over the past forty years, a new kind of method has increasingly been used in the social sciences: that of the computer simulation of social processes (Axelrod, 1997; Epstein \& Axtell, 1995; Gilbert \& Troitzsch, 2005). Computational sociology (or social simulation) is an outstanding method for modelling and building explanations of social processes, based on ideas about the emergence of complex behaviour from simple activities. With this technique we can study properties of emergent orders that arise from local interactions among a multitude of independent components. And we can understand the ways in which such emergent orders can influence or constrain the individual actions of those components. This process is known as 'self-organisation' and is characterised by the concepts of 'bottom-up' and 'downward causation'. 
However, despite its possibilities, which exceed the limits of traditional methods in the social sciences, social simulation also has some epistemological and methodological drawbacks. First, although the method is widely used in the field, there is no agreement about the idea of 'emergence' and its value for theory and explanation. Second, as a consequence, there is a division between 'individualist' and 'collectivist' emergentists. Finally, most research using social simulation ignores the importance of symbolic communication in the social realm, even though only this process can be called social in its own right. Because social communication is not an important issue within the mainstream of computational sociology, it is not hard to understand the reason why the most important advances in computational models about the emergence and the evolution of symbolic communication are restricted to computational linguistics ( $c f$ the review of Perfors, 2002).

In order to analyse how these drawbacks can be overcome, in section II we begin by examining the notion of emergence in order to show the ambiguity of the concept and draw out some relations between that notion and the different positions in computational sociology (section III). Then, we assess the over-ontological sense of emergence that both holistic and individualistic accounts of social reality propose and we delineate a possible alternative based on the concept of 'medium downward causation' (section IV). We relate this alternative to the distinction between individual and communication as advanced by Niklas Luhmann's theory of communication, because, we hold, this theoretical framework is well positioned to be a sociological support for developing social simulations (section V). We present that theory and conclude with a synthesis and some generalizations about the fruitful dialogue between social simulation and Luhmann's theory (section $\mathrm{VI})$.

\section{The two souls inhabiting emergence}

In all the discussions about emergent phenomena there are two constants (Schröder, 1998). First, emergent properties are always the properties of complex systems. So, elementary particles do not have emergent properties. Second, a property of a complex thing, in order to be emergent, must not be a property of a proper part of that thing. Thus, properties such as mass, velocity and charge are not emergent properties. These two restrictions are uncontroversial, because they say nothing about the possible relation between the parts of a thing and its emergent properties except that they must be at different levels. The quarrels arise when we try to define that relationship by answering two questions: (a) if we accept that there are elementary properties, can there be authentic emergent properties endowed with causal power? (b) if we recognise the micro and macro levels as independent, can the properties of the parts explain the emergent properties? These related ontological and epistemological topics are the focus of several debates.

In its strong sense, the concept of emergence means that nature and society articulate themselves on different levels of organisation, and that each of these levels yields its own novel causal powers which emanate from the emergent levels onto its constituents in the lower level (i.e., downward causation). Thus, the related concepts of emergence and downward causation presuppose that several levels of organisation coexist, be it merely as levels of description or as levels of description as well as ontology. These kinds of global organisations are called emergent because they cannot be reduced to the sum of the properties of their elements. In this way, for instance, consciousness is not deducible (therefore, it might be said, is not explainable) from neuronal properties alone and, similarly, society is not reducible to individual properties alone. In a more general way, no higher

level is explainable from its constituent units. Here, the motto is: 'the whole is more than the sum of 
its parts'. In order to explain that 'more than', the ontological claim about the existence of emergent properties is frequently followed by a kind of epistemological statement that asserts the nonreducibility of emergent (or macro) properties to properties of the parts in isolation.

This strong concept of emergence is often formulated in heavily metaphysical terms (for a similar and more extended claim, see Bitbol, 2007). Those who defend the concept of emergence want to know whether there truly exists such emergent large-scale properties and whether these properties are more than just epiphenomena; accordingly, they want to know whether they have or do not have the causal power of altering other (large-scale or micro-scale) properties.

This formulation of emergence is not surprising considering its historical origins. Alexander, Morgan and Broad, the classical emergentists (McLaughlin, 1992) invented and developed the concept during the early twentieth century in order to find a satisfactory compromise between two extreme ontologies: reductionist materialism and dualism. The first of these two ontologies is monist and materialist; it says that there exists, in the world, nothing else other than material elements and their properties. The second ontology is dualist; it says that there are two substances or two realms of being: mind and matter, life and inanimate matter, individuals and society. Classical emergentists tried to develop a middle stance between these two ontologies. Since then "[e]mergence has been perceived as a third path between dualism and identity theory, and this third path is generally known as nonreductive materialism." (Sawyer, 2002, p. 554). But being a middle path does not preclude it showing a bend towards one or the other of the two extremes, as Bitbol (2007) argues:

Emergentism comes very close to monist materialism if it takes the high level behavior as a superficial symptom, with no relevance whatsoever to the real physical processes taking place at the low level (...). Conversely, emergentism comes closer to dualism when it tries to endow the emergent properties with some sort of ontological consistence, and with causal powers of their own (p. 294)

These differences, related to the ontological and epistemological status of emergence, have been reinterpreted in sociology to link them with the longstanding debate over methodological individualism and methodological collectivism. The debate is based on the answer that social theorists make to the question: Where must social theory aim its attention in order to construct explanations about the social realm? There are two possible answers, namely, 'individual entities' (actors, individual action, desires, beliefs, etc.) or 'collectives entities' (institutions, norms, structures). This debate has been at the heart of social theory from its origins. For example, Emile Durkheim argued that social properties have causal force on the individual. His defining criterion of the social fact was its external constraint on the individual and, consequently, his methodological recommendation was that sociologists must consider the nature of society, not the nature of individuals (Durkheim, 1982, p. 63). However, some classical thinkers put forward the opposing claim. Max Weber proposed that social phenomena must be explained by showing how they result from individual actions, which in turn must be explained by reference to the intentional states that motivate the individual actors (Weber, 1978).

The concept of emergence was reinterpreted according to this long-lasting division inside sociological theory, concerned with the relationship between agency and structure, micro and macro. Consequently, existing sociological uses of emergence are contradictory. On the one hand, many accounts of the micro-to-macro link use the concept of emergence to argue that only individuals bring about the social regularities we observe (Coleman, 1964; Elster, 1989). On the other hand, 
methodological collectivists invoke the concept of emergence to indicate that, although individuals exist, collectives possess emergent properties that cannot be reduced to individual ones (Archer, 1995; Bhaskar, 1982; Luhmann, 1996). Sawyer talks about the 'slippery concept of emergence' in sociology and argues that "two opposed sociological paradigms both invoke the concept of emergence and draw opposed conclusions. The problem arises in part because sociologists have not developed an adequate account of emergence" (Sawyer, 2002, p. 552).

\section{Emergence and computational sociology}

In this context, what happens with computational sociology? As we shall explain, the uses of emergence in computational sociology are also ambiguous and unstable. Although the concept has become widely used, it continues to be vaguely defined and to stand in for different propositions about social generative mechanisms.

Social simulation is an excellent technique for modelling and understanding social processes, based on theories about the emergence of complex behaviour. The mainstream definition within the field establishes that a phenomenon is emergent when it can only be described using terms and measurements that are inappropriate or impossible to apply to the component units (Bankes, 2002; Gilbert, 1995; Squazzoni, 2008). Despite this epistemological notion of emergence, most researchers in the field recognise the affinity between the concept of emergence and social simulation. Gilbert and Troitzsch (2005) indicate that "the emphasis on processes and on relations between components, both of which can be examined by means of simulation, accounts for the developing link between this theoretical perspective and simulation research" (p. 12). Computational sociology provides experimental methods in order to investigate the emergence of social patterns from individual interactions and the possibility of using computer coding as a way of formalising dynamic social theories. Hence, for these authors, there is a strong link between the theoretical concept of emergence and social simulation.

Nevertheless, not everyone in computational sociology shares this opinion about the concept of emergence and its value as a theoretical support for computer modelling. Epstein (2007), another leader in the field of simulation, seems to look on it with suspicion. He doubts its practical usefulness: "I have always been uncomfortable with the vagueness and occasional mysticism surrounding this word" (p. 31). For him, the problem with emergentism resides in emergent phenomena being unexplainable in principle, because the parts cannot explain the whole. But Epstein (2007) indicates that

Obviously, "wholes" may have attributes or capabilities that their constituent parts cannot have (...) Equally obvious, the parts have to be hooked up right - or interact in specific, and perhaps complicated, ways - for the whole to exhibit those attributes. We at present may be able to explain why these specific relationships among parts eventuate in the stated attributes of wholes, and we may not. But, unlike classical emergentists, we do not preclude such explanation in principle (p. 36, italics in original)

Epstein puts forward the idea that it "is precisely the generative sufficiency of the parts that constitutes the whole's explanation" (Epstein, 2007, p. 36). This claim goes against emergentism, or, at least, it goes against classical emergentism. For Epstein the whole point in explaining a macroproperty is to uncover the rules that bring about that macro-property from the bottom-up. 
Consequently, for Epstein, agent-based modelling is reductionist par excellence. By attempting to generate social phenomena on computers or in mathematical models, "we are denying that they are unexplainable or undeducible in principle - we're trying to explain them precisely by figuring out microrules that will generate them" (Epstein, 2007, p. 36). Agent-based modelling is defined here in reductionist terms: because only agents and their local interactions are modelled, higher-level patterns must be epiphenomenal. The term 'emergent' is still employed by Epstein, but in his perspective it has a very restricted sense, meaning just "arising from decentralized bilateral agentinteractions" (Epstein, 1999, p. 49).

Although less radical in his methodological individualism, Hedström shares with Epstein similar epistemological grounds. He highlights the importance of constructing mechanism-based explanations, which implies describing how the social realm is brought about by individuals' actions and interactions. For him, because only individuals exist, modelling must consider their characteristics to explain the emergence of social structures. However, Hedström does not rule out the conditioning power of social structures over individuals. For him, "[s]ocial phenomena, as here defined, refer to properties of groups of individuals (...) These social phenomena are the result of individuals' actions, but they also causally influence individuals' actions” (Hedström, 2005, p. 70). In a more recent work, Hedström and Ylikoski explicitly reject the association between mechanismbased explanations and methodological individualism. According to them, mechanism-based explanations require some form of 'structural individualism', which suggests that although in principle all social facts might be explained in terms of individuals, relations and relational structures are important. Thus, "[f]acts about topologies of social networks; about distributions of beliefs, resources, or opportunities; and about institutional or informal rules and norms can have a significant role in mechanism-based explanations" (Hedström \& Ylikoski, 2010, p. 60). Consequently, computational sociology (specifically, agent-based modelling) is a "formalism designed for analyzing the relationship between individual-level and social-level phenomena, whatever these phenomena may be" (Hedström, 2005, p. 76).

The path followed by those who argue in favour of mechanism-based explanations in sociology in general and computational sociology in particular seems to be the most fruitful option. The result is that the previous line separating methodological individualism and holism in computational sociology becomes blurred and the two paradigms no longer appear as clear-cut opposites. As Squazzoni (2008) claims, "advocates of methodological and ontological individualism now seem more inclined to take into account institutions and social structures as macro constraints upon individual action" (p. 2). Thus, some emergent social structures are seen as the 'social situations' that at the same time constrain and make individual action possible. This account avoids the problematic commitments of dualism and reductionism. Macro properties are emergent in the sense that they are produced by individuals' interactions at the micro-level but, at the same time, those macro properties are not derivable from a single micro-level entity.

Consequently, to explain a social phenomenon means to identify a social mechanism: to make explicit a constellation of entities and activities that are organized such that they regularly bring about a specific type of outcome (Hedström, 2005; Hedström \& Bearman, 2011). Social simulation provides a method to falsify those proposed mechanisms. Using this modelling approach, the researcher builds an artificial society in which the mechanisms are translated as the model's microspecifications, that is to say, the set of behavioural and simple rules that specify how the agents behave and react to their environment (which includes other agents). Since agents' actions are not independent, feedback enters the system and a continuous interplay between the emergent structures 
and the agents' actions takes place, altering the dynamics of the system and moving it towards unpredictable states. When the simulation can generate the type of outcome to be explained, the researcher has provided a computational demonstration that a given microspecification is in fact sufficient to generate the macrostructure of interest (Epstein, 1999). This demonstration, called generative sufficiency, provides a candidate mechanism-based explanation of the macro-phenomenon. The researcher can then use relevant data and statistics to test the agreement between the 'real world' and the generated macrostructures in the computer. Once the mechanism has proven to be sufficient to generate the phenomenon of interest in silico and it has been tested against real-world data, the researcher has established a valid explanation of the social process. On the other hand, if the model cannot generate the outcome to be explained, the microspecification is not a candidate explanation of the phenomenon and the researcher has demonstrated the hypothesised mechanism to be false.

\section{Emergence and downward causation}

Individualist emergentism leads to understanding emergent properties as epiphenomenal (without causal power) because only individuals' actions create those properties; this is the main difference from those who assert that social properties are not deducible from the isolated individual. However, this 'ontological individualism' does not imply the inevitability of 'methodological individualism'. The logical error of making ontological arguments when supporting methodological claims is common in the philosophy of the social sciences. As Sawyer (2002) notes, the fact that social properties are nothing more than their individual supervening base does not entail that an explanation must be provided in the language used to describe individuals. Moreover, consistent with epistemological empiricism, micro-sociology asserts that valid sociological explanations cannot be structural, but must always refer to situational micro-dynamics such as actors' desires, beliefs or opportunities because only individuals, not structures, exist and are endowed with causal powers. However, we could just as plausibly say that because the notion of 'individuality' is a theoretical and by no means well-clarified - abstraction, individuals do not 'act' in any more realistic or empirical sense than do structures. As Fuchs (1989, p. 178) wonders, "[c]ausal explanations are attempts at making sense, making sense requires languages appropriate for particular analytical purposes, but why should there be only one language (that of microsociology) into which all our accounts must be translated to make them 'more empirical' and 'causally stronger'?"

Those who believe in the causal power of emergent properties fail in a similar way. Most affirm the ontological status of emergent properties by referring to the non-linearity of the equations ruling elementary processes. The key point is that the complex behaviour of non-linear systems cannot be predicted from the initial state of the components, experimentally measured with limited accuracy. This is taken by some to mean that there is really more in the global behaviour than in the individual processes. But, if we want to prove the real existence of emergent properties, endowed with causal power, we cannot be satisfied with showing that complex systems are unpredictable in practice because of restricted knowledge of the initial conditions and the underlying deterministic laws. This only yields epistemological emergence, not ontological emergence (Bitbol, 2007; Schröder, 1998). Furthermore, the very concept of 'downward causation' seems to take for granted the definition of 'causation' and what is to be 'caused' (where to cause could be to restrain, to structure, to determine, to govern, or to delimit future events). The underlying problem is that nobody really knows what is meant by 'causation', 'cause' or 'causing' (Hulswit, 2005). 
Therefore, we have one concept and at the same time, one dilemma. The main question is whether we can define the concepts of emergence and downward causation in a productive way for sociological research. Emmeche and colleagues (2000) argue that an emergent property can be framed in a materialist and evolutionary perspective. To do so, the relation between levels must be inclusive, permitting the existence of different ontologies, all within the physical level and without violating the physical laws of the lower levels. Thus, a property of a complex system is said "to be emergent just in case, although it arises out of the properties and relations characterizing simpler constituents, it is neither predictable from, nor reducible to, these lower-level characteristics" (Emmeche et al., 2000, p. 14). As a kind of immediate extrapolation of this idea, downward causation is usually employed in the philosophy of science as a designation for an alleged top-down effect, which emanates from the emergent properties onto their constituents in the lower level.

According to Emmeche and colleagues, in the scientific and philosophical literature there are three different conceptions of downward causation, based on different ontological theses about inter-level relations and types of causation. They call these three conceptions, respectively, Strong, Medium and Weak downward causation (Emmeche et al., 2000, p. 24). Whereas strong downward causation is presented as some kind of downward efficient causation, medium and weak downward causation are two different versions of downward formal causation.

They characterise strong downward causation by (1) a sharp distinction between the higher and the lower level of phenomena, each of which is constituted by qualitatively different kinds of substances, and (2) the fact that higher-level substances exert an efficient causal influence on substances at a lower level. Even though the higher-level entities consist of lower level entities, they "possess a substantial existence qualitatively different from lower level entities" (Emmeche et al., 2000, p. 19). Moreover, strong downward causation implies a 'substance dualism'. Thus, for instance, to emphasise the specifically biological character of a living cell emerging from its physico-chemical basis, one often describes the cell, more or less implicitly, as a living substance that efficiently causes changes in the interactions between its physico-chemical components. But this position is, according to these authors, irrational. The mistake consists in presupposing that the cell and biochemical processes can exist independently of one another and that there can be a causal relationship between them, for the biochemical processes do not cause but constitute the cell. For this reason Emmeche and colleagues reject this strong version of downward causation, and argue that it involves a revival of the vitalist fallacy: "the identification between this temporal chain and the constitutive relation" (Emmeche et al., 2000, p. 19). As a scientific concept, strong downward causation should be rejected.

The concept of medium downward causation, as proposed by Emmeche and colleagues (2000) might be a good first step in order to overcome the many problems posed by strong downward causation. The specific characteristic of medium downward causation is that downward formal causation is understood in terms of constraining conditions and part-whole relationships. They define medium downward causation as follows: "an entity on a higher level comes into being through a realization of one amongst several possible states on the lower level - with the previous states of the higher level as the factor of selection" (Emmeche et al., 2000, p. 24). They maintain that "bigher level entities are constraining conditions for the emergent activity of lower levels. And (...) in a process, the already realized higher level states are constraining conditions for the coming states.” (2000, p. 25; italics in original). Contrary to strong downward causation, "it does not allow higher level phenomena to have a direct influence on lower level laws". This definition entails not only that the higher level constrains the activity on the lower level, but that it also constrains "which higher level phenomenon will result 
from a given lower level state." Moreover, according to medium downward causation "the same lower level constituents may correspond to a series of different higher level phenomena" (Emmeche et al., 2000, p. 25).

Finally, weak downward causation is the third sense that downward causation can acquire. Emmeche and colleagues define this weak version as follows: "the higher level is conceived as an organizational level, characterized by the organization, the whole, the pattern, the structure, in short the form into which the constituents are arranged." (Emmeche et al., 2000, p. 26). This weak version differs in at least four important aspects from medium downward causation: (1) the higher level is not seen as a substance, but as an organisational level - the structure or form according to which the lower level entities are organised; (2) the formal cause in weak downward causation may be understood by analogy to the concept of 'stable attractor.' A stable attractor is a kind of steady state - a bounded set of points in phase space - in a dynamical system. Depending on the initial conditions, the dynamical system may evolve in the direction of one attractor; (3) the 'downward causal influence' is not understood in terms of constraining conditions, but static and timeless conditions which are not the result of the very process of emergence (as in medium downward causation); (4) the same lower level phenomenon does not correspond to several higher level phenomena: depending on the initial state, a dynamic system evolves in the direction of one specific attractor, which itself does not evolve (Emmeche et al., 2000, p. 26).

Although Emmeche and colleagues admit that both medium and weak downward causation are the only two viable candidates for a 'rational account' of causation, the latter implies a more structural approach that does not consider the attractors of any product of creation or contingent historical process. Instead, in medium downward causation the attractors are created only in the process in which the higher-level properties emerge (Emmeche et al., 2000; Hulswit, 2005). Attractors, either chaotic or stable, were developed to analyse dynamical systems formalised by differential or difference equations, so they are mainly used in mathematical modelling (Brown, 1995; Gleick, 1988). Social simulation, and specially agent-based modelling, aims to uncover the underlying generative mechanisms that bring about the observed regularities at the macro level, so in principle any steady state that the system reaches can be explained by these mechanisms and the contingent and temporal dynamics they produce (Manzo, 2007; Squazzoni, 2008). Additionally, medium downward causation establishes causality as constraining conditions, which is an appealing idea in sociology, as different sociologists have understood the micro-macro link (Archer, 1995; Coleman, 1964; Elder-Vass, 2005; Luhmann, 1996). We shall return to this point later.

However, despite being a fruitful concept for social simulation and sociology, medium downward causation, as defined by Emmeche and colleagues, still poses some important problems. Firstly, the authors are ambiguous regarding the type of causality that this concept entails. According to them, "the entities at various levels may enter part-whole relations (e.g., mental phenomena control their component neural and biophysical sub-elements), in which the control of the part by the whole can be seen as a kind of functional (teleological) causation, which is based on efficient, material as well as formal causation in a multinested system of constraints" (Emmeche et al., 2000, p. 25). However, the authors never clarify in what sense this is teleological rather than efficient causation, nor do they tell us how this kind of teleological causation is related to formal, efficient or material causation. Secondly, the authors reject "any untenable metaphysical idea of a temporal causal process from a higher level to a lower one (or vice versa, for that matter)" (Emmeche et al., 2000, p. 32). But this is a questionable conception of causality. As the authors claim, medium downward causation implies that an emergent property of a higher-level entity is not caused by its parts or by their powers, as the 
concept of efficient causality implies. As we shall explain latter, symbolic communication does not cause but constitutes the social realm. However, when one wants to describe the causal power of any higher-level property onto its components, the temporal dimension cannot be ruled out. The scientific idea of causation connotes transitivity (Hulswit, 2005; Kim, 2001), that is, the cause precedes the effect.

Borrowing a distinction proposed by Elder-Vass (2005), we can explain the relationship between emergence and downward causation avoiding the ambiguities of Emmeche and colleagues' proposal. We shall understand emergence as a synchronic relationship between a whole and its parts, whereas downward causation should be understood as a diachronic relation in which the powers of a group of entities, at one moment, causally determine the events that follow at the next. As Elder-Vass claims, "emergence, then, is a synchronic relation amongst the parts of an entity that gives the entity as a whole the ability to have a particular (diachronic) [and top-down] causal impact." (Elder-Vass, 2005, p. 321). Consequently, we can modify the definition of medium downward causation by adding the requirement, common in complex systems, of 'efficient causality restriction' (Brodu, 2009), which includes a temporal restriction. The higher-level entities cannot modify lower system laws, but can only constrain (although not rule out completely) future possibilities. Thus, higher properties restrict the multiple possibilities that lower entities have, and by doing so, also constrain which higher-level phenomena will result from that given lower level. In this scheme, downward and upward causation are the co-limitation of possibilities over time between the micro and the macro levels.

In contemporary sociological theory, critical realism has thematised the concept of emergence in a similar way. Like Emmeche and colleagues, Archer stresses the autonomy of both individual actions and the social structure, and the continuous cycle of interaction between them. Archer's morphogenetic approach is based on two propositions: "that structure necessarily pre-dates the action(s) which transform it; and that structural elaboration necessarily post-dates those actions" (Archer, 1995, p. 76). The methodological consequence of this separability between the micro and the macro, similarly to the idea of 'medium downward causation' discussed above, is that it enables social researchers to analyse the ongoing interplay between them while still insisting on the ways the two levels are related and co-evolve over time.

However, as many other authors have noticed (Elder-Vass, 2007a; Hedström, 2005; Sawyer, 2002), Archer's morphogenetic approach has internal ambiguities and inconsistencies, especially in relation to the concept of emergence, which oscillates between 'analytical dualism', on the one hand, and the insistence that emergent structures have real 'causal powers' (although mediated through 'social agency'), on the other. Furthermore, Archer (1995, p. 183) claims that it is emergence over time that endows social structures with causal powers and, consequently, makes them real. In her perspective, current social structures emerged from the past actions of individuals, such that they cannot be explanatorily reduced to the actions of current individuals. But this position is problematic. Emergence over time does not provide an ontological argument for social causation, because although social structures require past actions to be stabilised, they also need - if they come about to be instantiated by contemporary actions or agents. As Sawyer (2002, p. 570) observes, "[i]n an artificial society simulation, structure emerges over time, but can only continue to exist through persisting interactions among elements".

The problem with this approach is whether emergent properties can constitute their own substances. The ontological claim that a higher entity "is a real substantial phenomenon in its own right" (Emmeche et al., 2000, p. 23) and that it can bear 'causal powers' (Archer, 1995, p. 195) becomes 
ambiguous because the implicit concept of downward causation in these perspectives entails a shift from a description in terms of substances to a description in terms of interactions. But from a sociological standpoint, the main issue is whether sociological observers can distinguish the social realm as different from and not conflated with the individual one. In the following section, we introduce a sociological alternative to the individualistic and holistic interpretations of social phenomena. This alternative is based on the structural coupling of individual consciousness and social communication as advanced by Luhmann's theory of self-referential social systems.

Our attempt to integrate self-referential social systems theory with the concept of emergence must, however, be qualified in advance, for Luhmann underestimates the value of the concept in several parts of his work. According to Elder-Vass (2007b), in designating a system as autopoietic, the German sociologist is in fact rejecting any emergentist view of the social order. Thus, even though a social system requires individuals, their influence can be ignored for the purpose of explanation, since everything that is used by the system in its reproduction is produced by the system itself.

Whether the unity of an element should be explained as emergence 'from below' or as constitution 'from above' seems to be a matter of theoretical dispute. We opt decisively for the latter. Elements are elements only for the system that employs them as units and they are such only through this system. This is formulated in the concept of autopoiesis (Luhmann, 1996, p. 22).

This claim could prima facie undermine any attempt to link Luhmann's theory to the concept of emergence, since the latter recognises the importance of the dynamics that, 'from the bottom', bring about the macro patterns we observe at the system level. For Luhmann, instead, it is the system itself that defines in a top-down fashion what is possible and what is not within its boundaries. In this perspective, either the system exists or does not exist; if it does exist, then it is an autopoietic system describing strong downward causation. And yet Luhmann sometimes slips into the excluded zone between being and not being. For instance, it is said that "[o]nce art becomes autonomous, the emphasis shifts from hetero-reference to self-reference" (Luhmann, 2000, p. 149). Art - as an autopoietic system - is or is not, and art - as an evolutionary system - becomes. Therefore, a closer scrutiny of Luhmann's work reveals a clear tension between, on the one hand, his binary rhetoric that leads him to claim the absolute autonomy (i.e., operative closure; "the thesis of operative closure says merely that for any system, only what is accessible for its own operations is accessible; that, therefore, for any system, only what can be formed as a unity through these operations can be a unity", Luhmann, 1988, p. 339) of the systems and, on the other, his evolutionary perspective that leads him to recognise that social systems are the result of a continuous process of differentiation from their environments.

To explore this issue in more detail is beyond the scope of this article, but the important point is that, as Winthrop-Young (2003) claimed, these two logics remain incompatible in Luhmann's work. When the autopoiesis of the social systems is stressed, Luhmann meets what Archer (1988) identified as a 'downward conflation fallacy': a sociological determinism that elides individual agency in favour of the causal powers of the social system. However, when Luhmann addresses the evolutionary differentiation and stabilization of social structures, his theory becomes closer to the emergentist view. The key concept is that of communication. Communication is an emergent order brought about by the situation of 'double contingency' immanent in social relations. Over time, the emergence of communication exerts structural limitations on the possibilities of individual selections and it internally differentiates into meaningful constellations of co-ordinated selectivity (i.e., social 
systems such as economy, politics, science). In turn, these social systems bring about different properties at the lower level: different expectations that might be confirmed or disappointed. The theory of communication that Luhmann developed emphasises the dynamic interplay between psychic systems (i.e., individuals) and social structures. Therefore, it might be said that in Luhmann's theory the micro-macro relation is framed, avant la lettre, as medium downward causation. For these reasons we claim that his communication theory can be linked to an emergentist explanation of the social order. And consequently, his theory is well positioned to inform computational models of social processes.

\section{Social communication and social emergence}

As Luhmann (1990, p. 6) says, "[c]onfronted with the question of elementary units, most sociologists would come up with the answer: action. Sometimes 'roles' or even human individuals are preferred". These traditional options overlook both the importance of communication and its relation with the concept of emergence. Sawyer (2005) has described the issue in the following terms:

A theory of social emergence requires an explicit theorization of symbolic communication and dynamic processes. Yet for the most part, sociological theorists who focus on the micro-macro link have not theorized communication, nor the role that communication plays in micro-macro relations (p. 187)

In order to explain the emergence of society from individuals, Sawyer makes a basic assertion: the emergence of language is the emergence of society. By analyzing three broad classes of artificial societies, namely reactive, cognitive and collaborative agent societies, Sawyer suggests that differences in communication result in different emergent processes and outcomes. Emergence occurs only when there is an interaction among agents, but what is more important, "different collective properties emerge and the processes of their emergence are different when the agent communication language is changed" (Sawyer, 2005, p. 188). Thus, Sawyer concludes that (a) the model of communication that is used in a society has causal consequences for the type of emergent regularities under study and (b) a theory of symbolic communication must be a core component in any explanation of social phenomena.

Nevertheless, Sawyer's theory of social emergence, which is based both on collaborative activities among agents and the distinction between ephemeral and stable emergence (Sawyer, 2005), is insufficient to explain the evolutionary stabilisation of social structures and the emergence of generalised symbolic media such as money and power. This does not mean that language is not important or that it does not have any role in social analysis. Quite the opposite, language allows the structural coupling between the social realm and individual thinking (Baraldi, 1993). According to Maturana and Varela (1979), who defined the concept, structural coupling refers to situations in which there is a history of recurrent interactions leading to congruence between two or more systems. Language, as a communication media, is at individuals' disposal to transform thoughts, or any psychic state, into words that may be understood or misunderstood by other individuals. But although important, language does not exhaust social reality, as it seems to be claimed by Sawyer. Language is not enough to stabilise complex social orders because it gives no motivation for coordinated selection among individuals - other individuals can always disagree. As Mascareño (2008) argues, "[language] can limit selection possibilities but motivation itself derives from the structured 
expectations of meaningful constellations allowed by the evolution of symbolically generalized communication media" (p. 203). The mistake is then to deposit into language more than language can actually hold. We need to use another theory about the emergence and evolution of social communication.

In the Parsonian tradition, ego and alter are each objects of orientation for the other. Every social interaction constitutes a situation of double contingency, since both ego and alter know that both know that one could also act differently (Vanderstraeten, 2002). The concept of double contingency implies that ego's gratifications are contingent on alter's selection of action and, in turn, alter's reaction will be contingent on ego's selection resulting from a complementary selection on alter's part and so on (Parsons \& Shils, 2001). And for both alter and ego there are unlimited possible selections. Luhmann follows this conceptualisation and agrees with Parsons that social order is impossible unless the problem of double contingency is solved. In Luhmann's words: "We would emphasize that the problem of double contingency belongs to the conditions of possibility for action and that (...) actions can be constituted only in these systems and only by solving the problem of double contingency" (Luhmann, 1996, p. 104). Luhmann explains the connection with the emergence of social order in the following terms:

The basic situation of double contingency is then simple: two black boxes, by whatever accident [sic], come to have dealings with one another. (...) For the few aspects through which they deal with one another, their capacity for processing information can suffice. They remain separate; they do not merge (...) They concentrate on what they can observe as input and output in the other as a system in an environment (...) They can try to influence what they observe by their own action and can learn further from the feedback. In this way, an emergent order can arise that is conditioned by the complexity of the systems that make possible but that does not depend on this complexity's being calculated or controlled. We call this emergent order a social system (Luhmann, 1996, p. 110; italics in original).

To overcome the problem of double contingency is to produce an emergent order, 'independent' of both alter and ego. But, if social theory cannot explain the stability of social order on the basis of norms or values, what is to take their place? Because double contingency is a pre-eminently social problem, the solution requires the use of a pre-eminently social operation; namely communication. Social order can only be produced by means of communication, although it is this order that also enables communication (Vanderstraeten, 2002). This is a counterintuitive proposition. A commonsense perspective would maintain not only that communication must necessarily be between individuals, but also that only individuals are able to communicate. Contrary to this view, Luhmann argues that, fundamentally speaking, individuals cannot communicate at all, not even in their capacity as psychic systems. In Luhmann's theory, individuals (or psychic systems) operate in terms of meaning in the form of a closed connection of consciousness. Similarly, social systems operate in terms of meaning in the form of a closed connection of communication. Therefore, the strong axiom of the theory is: communication alone is able to communicate (Andersen, 2003; Luhmann, 2007). Society is autopoietic from this perspective, because it is understood as an emergent and selfreferential phenomenon that cannot be reduced to something other than itself, neither to consciousness nor to a sum of individuals' actions. Here we see the phenomenological insistence on observing society as it appears without reference to conditions external to society. And individuals, as psychic systems, are external to society. 
But, what is communication? Communication is a shared actualisation of meaning that happens when alter utters and ego understands, and this is performed only by the unity of these two different selections. It is necessary both that something be said by someone (alter) and that someone (ego) understands what is said. Alter's utterance and ego's understanding form a 'unity' because they give reality to communication only together: communication exists only if alter utters and ego understands. As explained by Baraldi (1993), if alter says, 'It is too late, I must go', communication is produced by ego's understanding of both alter's motives (e.g., she is in a hurry, is bored, has an important appointment, wants ego to say 'stay') and the information, 'It is too late, I must go'. The relevance of understanding motives comes from the necessity to attribute responsibility for the utterance. This is a necessary condition for communication. Without such an attribution, ego cannot refer to alter's utterance, and then communication is not realised. There is only perception: information attributed to something that has no motives or responsibility for it (e.g., a watch displaying the time or an engine roaring in an unusual way).

Communication is an emergent order, a state sui generis. It emerges through a synthesis of three selections, namely: alter selects information from a horizon of meaningful possibilities; she or he instantiates it through language or actions (utterances); ego observes alter's conduct and understands or misunderstands this utterance and its information (Luhmann, 1990). A psychic system can neither produce nor control communication because it can neither produce nor control the unity of selection of information, utterance and understanding (i.e., a communicative event). As the social dimension implies a double perspective (ego and alter ego), in the single event of communication each individual either utters or understands: she cannot do both things. If alter utters 'It is too late, I must go', she can understand an utterance of information only in the following communicative event. Therefore, the unity of a single communication happens outside the psychic domain, as the unity of the triple selection we mentioned before (and for this reason, individuals, as psychic systems, cannot communicate).

Of course, ego can accept or reject the offer, but anyway it might be said that ego understands alter's proposal - misunderstanding is always a form of understanding. "[T] he acceptance or rejection of an expected and understood selection are not part of the communicative event; they are connected acts (...) Viewed dynamically, the unity of an individual communication is merely its connectivity" (Luhmann, 1996, p. 148). For this reason, for Luhmann, "understanding always includes misunderstanding, and if one does not add on presuppositions, the component of misunderstanding becomes so great that the continuation of communication becomes improbable" (Luhmann, 1996, p. 158). Thus, the communication theory Luhmann is trying to formulate starts from the premise that it is implausible. And because the basic element of society is communication (and not individuals), social order also appears as a highly improbable event.

This improbability of which we have become unaware must first be understood, and to do so requires what might be described as a contra-phenomenological effort, viewing communication not as a phenomenon but as a problem; thus, instead of looking for the most appropriate concept to cover the facts, we must first ask how communication is possible at all (Luhmann, 1990, p. 87)

Luhmann (1990) argues that communication - if it comes about - must overcome three obstacles or improbabilities: (a) the individuality of human consciousness, (b) the extension of communication beyond direct participants and (c) the improbability of success. The first improbability is related with understanding; given that their bodies and minds are separate and individual, it is unlikely that one 
person can understand what another person means. Meaning can be understood only in context, and for each individual consists primarily of what their own memory supplies. The second improbability is related to the spatial and temporary limitations of communication in reaching recipients. That is, it is improbable that a communication can get to more people than are present in a given situation. Even if the communication finds a means of conveyance that is constant over time, it is still unlikely that it might attract attention: in other situations people have other things to do. The third improbability is that the communication, even if it is understood, is accepted and followed. By success Luhmann means "that the recipient of the communication accepts the selective content of the communication (the information) as a premise of his own behaviour, thus joining further selections to the primary selection and reinforcing its selectivity in the processes" (Luhmann, 1990, p. 88). Communicative success is the coupling between alter's expectations and ego's selections. Regarding all these three improbabilities, Luhmann (Luhmann, 1996, p. 159) argues that "no social system can be formed without communication. (...) The immanent improbabilities of the communicative process and the way in which they are overcome and transformed into probabilities regulate the construction of social systems".

Despite these improbabilities, social order exists and we communicate daily. This is because social evolution has solved these improbabilities with three consequent mechanisms, which Luhmann identifies with the concept of media. The first evolutionary achievement, to overcome the first improbability mentioned above, is language. Language is a medium that, via acoustical and optical signs, makes it more probable that ego understands alter. It can, through the use of equivalent signs, reinforce the impression that ego and alter hold equivalent opinions (Luhmann, 1990). Dissemination media, such as writing, printing and electronic broadcasting, contribute to expanding communication themes beyond the restrictive boundaries of interaction systems. These media immensely increase the scope of communication and result in a social order decoupled from local contexts and from idiosyncratic interpretations. However, according to Luhmann, both language and dissemination media make it even more doubtful which communications will succeed: by understanding, ego has more reasons to reject alter's communicative proposal; dissemination media do not allow direct questioning or instantaneous requests for clarification.

Language and dissemination media are preconditions to other media that make success probable, namely, symbolically generalised communication media. Only the latter media achieve the ultimate aim of communication: to motivate heterogeneous individuals to act and experience in a relatively coordinated way. They make possible, at the individual level, what Luhmann calls "the nexus between selection and motivation" (Luhmann, 1996, p. 161). And at the macro level, they make possible the emergence of meaningful constellations of co-ordinated selectivity that provide common significance, identifiable themes and complementary expectations - what Luhmann calls 'social systems'. Motivation is implied in the selection of symbolic media as they generate their own conditions of acceptability and diffusion. Thus, the multiple possibilities of selection that individuals have can be restricted, making social order more likely. The complexity of the social realm emerges through the reduction of these multiple possibilities and through the selective conditioning of this reduction. Symbolically generalised communication media achieve this by defining the limits of what is structurally possible in each case, that is, in each social constellation of meaning. Luhmann analysed multiple examples of such media: scientific truth within the scientific system (Luhmann, 1994a), power within politics (Luhmann, 1994b), validity within the legal system (Luhmann, 2004), love within intimacy (Luhmann, 1999) and beauty within art (Luhmann, 2000). 
Following Mascareño (2008), the social dynamic or micro-macro link thus produced can be explained in the following terms: In an upward direction, the process of mutual references from one individual (alter) to another (ego) continuously recreates the social order as stabilised constellations of meaning. Conversely, in a downward direction, these relatively stabilised structures of communication exert a conditioning effect on the progression of communicative events, which can be seen as a (medium) downward causation process expanding from the social, down to the psychic system. Alter's and ego's experiences and actions are modulated and coupled with stabilised expectations in social evolution. But society does not cover the whole spectrum of individual selectivity. The structural couple between individual and society does not mean that the latter determines the former, because this would break the operational closure of both individual and society. Rather,

[symbolic media only] motivates to follow the orientation given by stabilised systemic structures. Otherwise individuals would lose their acceptance capability for selections, even though their counterfactual behaviour can trigger variations in the constellations of meaning that move society in unpredictable and contingent directions (Mascareño, 2008, p. 6)

Symbolic media allow the emergence of structures of expectations that alter and ego use to reduce the uncertainty of their own double contingency; only with those media can individuals accept the selective content of the communication as a premise of their own behaviour. Symbolic media promote some selections and exclude others depending on the context of their instantiation: they motivate awareness of the other in intimate relations (love); payment operations in economic transactions (money); evaluation of the electoral consequences of political decisions (power); and the validity of arguments in science (scientific truth). Conversely, in each constellation of expectations, other selections are excluded: consumers generally do not buy in the supermarket using love; politicians who make decisions because they have been paid are considered to be corrupt; and even the Prime Minister must prove the validity of his arguments if he wants to submit a paper to a scientific journal. Therefore, different meaningful constellations bring about different expected selections, which might be confirmed or disappointed by the speakers at any time and at any place.

\section{Luhmann's theory and computational sociology}

In scientific research, the quality assessment of a theoretical approach does not come from some $a$ priori standard but rests on the description of the way research is actually conducted by the scientific community (Ahrweiler \& Gilbert, 2005). In this sense, the actual evaluation of a theoretical approach comes from answers to questions such as: do others accept this framework as being coherent with existing knowledge? Do other scientists use it to support their findings, inform their models or inspire their own investigations? Although still incipient, Luhmann's theory has received increasing interest from the social simulation community during the last few years. The German volume "Luhmann Modelliert" edited by Thomas Kron (2002) was the first systematic effort to show the usefulness of this theoretical framework for computational sociology. Another good example is Fleischmann's (2005) computational model of a simple Luhmann economy. Using an agent-based model, the author reproduces the evolutionary dynamic of the economy. For Luhmann, the circulation of the economy is made possible at the historical moment when economic institutions circumvent successfully the paradox of scarcity, according to which the richer supply of one is the greater need of the other (more abstractly, every access to scarce goods which serves the lessening of 
scarcity increases scarcity). Fleischmann's agent-based model showed conformity to Luhmann's hypothesis: giving specific initial conditions, the economy produces unevenness from unevenness.

In ecological modelling, Grant et al. (2002) draw upon Luhmann's theory to suggest a framework for quantitative modelling of coupled natural/human systems, which they use to simulate the effect of societal constraints on environmental action. They explore the usefulness of Luhmann's theoretical framework in establishing a structural linkage between social and biophysical models to represent quantitatively how society might relate to its environment, and how this interaction might influence specific environmental actions. To this end, the authors coupled this model with a simple ecological model that simulates use of a common forage resource by animals placed on a commons by two individual ranchers. The resulting simulations demonstrate the classic 'tragedy of the commons' (i.e., ranchers optimized their profits by increasing the number of animals on the commons nearly every year, forage resources were depleted, and market weights of animals decreased). Consistent with Luhmann's theory, society remained oblivious to environmental degradation until the environment sent a signal that could be translated into the code of one of its subsystems: when forage levels fell below a politically determined threshold, they were communicated as illegal. Society then took environmental action by mandating the removal of all livestock from the commons until forage levels recovered. Others authors have investigated, by using computer simulations, the relation between individual expectations and meaning (e.g., Duong \& Grefenstette, 2005; Fleischmann, 2005; Grant et al., 2002; Kron, 2002; Leydesdorff, 2005).

Recently, Marchione et al. (2010) developed an agent-based model to study the properties of an emergent communication system, in which groups of artificial and 'speechless' agents create a lexicon and spread it throughout the whole population of agents. In this model, a communicative event (dubbed 'a chatty game') happens following the three selections Luhmann identified in his theory: an agent is randomly selected as 'speaker', the agent refers to some object in the world, and it utters some cultural sign or 'word' (either a just invented one or one learned in previous interactions). Then, hearers understand (or misunderstand) the speaker's utterance by distinguishing between the uttered sign and its information (some object in the world). These communicative events happen repeatedly. The model results indicate that, when the agents confront an uncertain situation of 'double contingency' - that is, they never have direct access to each other's meanings or ontologies - a shared lexicon can emerge, on the condition that a group of agents develops a communicative strategy that favours their mutual understanding and allows them to reach more recipients for their utterances. This property is consistent with Luhmann's theory, since the first group of agents that overcomes these two improbabilities of communication (i.e., understanding and reaching recipients) is able to spread its own communication system. Moreover, Marchione et al. reported that the agents capable of spreading their lexicon were also 'immune' to external influence, a trait that has been identified in other social domains by similar computational and analytical methods (Xie et al., 2011). Therefore, there are possible and fruitful links between Luhmann's communication theory and current research in computational sociology.

\section{Conclusion}

In this paper we have considered some epistemological drawbacks to the concepts of emergence and downward causation. Many of them are based on the heavily ontological definition of emergent properties, which tends to split sociologists between those holding to an individualistic approach 
and those affirming a holistic one. In order to overcome these drawbacks, we have discussed some current trends in the philosophy of science, in which the concept of emergence is reconstructed with an understanding of causation in terms of constraining conditions and micro-macro relationships. We have argued that Luhmann's theory of self-referential social systems is consistent with such a stance on emergence and can deliver new insights. His theory defines social communication as an emergent phenomenon that makes possible the restriction of the unlimited possibilities of selection that individuals have. We have also paid attention to symbolically generalised communication media, which, at the lower level, make probable that individuals accept the selective content of the communication as a premise of their own behaviour and, at the higher-level, bring about constellations of common significance. We described those constellations as structures of expectations that alter and ego use to reduce the uncertainty of their own contingency.

This increasing attention to Luhmann's theory by the social simulation community is not hard to understand. His theory shares grounds with social simulation, such as second-order cybernetics, systems theory and complexity theory (Castellani \& Hafferty, 2009). But more important is that, as we have claimed in this paper, in Luhmann's theory society emerges from the bottom-up as communication and, over time, it limits the possible selections that individuals have. In practice, this conceptualisation is hard to distinguish from artificial society models of emergence processes. The communication theory advanced by Luhmann is coherent with the type of mechanism-based explanations that computational sociology translates into artificial societies. Given these common grounds and equivalent premises, the interconnections between Luhmann's theory and social simulation research are almost natural. Furthermore, his theory of communication, expressed in several works, describes in detail the logic inherent in each social system and the process by which social structures emerge. Luhmann's premises still pose, however, some challenges to computational sociology. For example, the theory of symbolic media can be considered, in Lakatos's terms, as a 'research programme' in its own right (Chernilo, 2002), because it explains the historical processes by which the constellations of co-ordinated selectivity become differentiated - therefore, this theory has empirical content and some of this content has been verified. However, there are no computational models of these evolutionary processes. This is a major topic for computational sociology, because it is accustomed to deal with the dynamic stabilization of simulated social structures.

Whether or not a social property can be reducible to individual properties is an empirical question that can only be resolved through empirical research. We hypothesise that the type of emergent properties that are inherent in the social realm are likely to result from the unique fact that the participating entities are symbolic agents. Therefore, what is needed to carry out such research is a reliable method by which those emergent properties can be generated and analysed. In short, social scientists require appropriate tools. Of course, this is not a defence of a mathematical or computational 'imperialism' in sociology. Throughout the short life of computational sociology it has been repeated that simulation should never be allowed to take command over sociological theorising (Cederman, 2005; Coleman, 1964). The growing number of artificial societies that have been informed by Luhmann's communication theory is sufficient to demonstrate the fruitful dialogue between social theory and computational models of social processes.

Mauricio Salgado (Corresponding Author)

Mauricio.Salgado@uab.cat

GSADI: Analytical Sociology and Institutional Design Group 
Department of Sociology

Universitat Autònoma de Barcelona, B Building Campus UAB, 08193 Bellaterra, Spain.

Nigel Gilbert

n.gilbert@surrey.ac.uk

CRESS: Centre for Research in Social Simulation

Department of Sociology

University of Surrey, Guildford, Surrey, GU2 7XH, United Kingdom.

\section{References}

Ahrweiler, P., \& Gilbert, N. (2005). Caffè Nero: the Evaluation of Social Simulation. Journal of Artificial Societies and Social Simulation, 8(4), 14. Retrieved from http://jasss.soc.surrey.ac.uk/8/4/14.html

Andersen, N. A. (2003). Discursive Analytical Strategies: Understanding Foucault, Koselleck, Laclau, Luhmann. Glasgow: Policy Press.

Archer, M. S. (1988). Culture and Agency: The Place of Culture in Social Theory (2nd ed.). Cambridge: Cambridge University Press.

Archer, M. S. (1995). Realist Social Theory: The Morphogenetic Approach. Cambridge: Cambridge University Press.

Axelrod, R. (1997). Advancing the art of simulation in the social sciences. Complexity, 3(2), 1622.

Bankes, S. C. (2002). Agent-based modeling: A revolution? Proceedings of the National Academy of Sciences, 99(90003), 7199-7200.

Baraldi, C. (1993). Structural Coupling: Simultaneity and Difference Between Communication and Thought. Communication Theory, 3(2), 112-129.

Bhaskar, R. (1982). Emergence, explanation and emancipation. In P. F. Secord (Ed.), Explaining Human Behavior: Consciousness, Human Action and Social Structure (pp. 275-310). London: Sage Press.

Bitbol, M. (2007). Ontology, matter and emergence. Phenomenology and the Cognitive Sciences, 6(3), 293-307.

Brodu, N. (2009). A synthesis and a practical approach to complex systems. Complexity, 15(1), 36-60.

Brown, C. (1995). Chaos and Catastrophe Theories: Nonlinear Modeling in the Social Sciences: 107. California: Sage Publications, Inc.

Castellani, B., \& Hafferty, F. W. (2009). Sociology and Complexity Science. Berlin, Heidelberg: Springer.

Cederman, L.-E. (2005). Computational Models of Social Forms: Advancing Generative Process Theory. American Journal of Sociology, 110(4), 864-893.

Chernilo, D. (2002). The theorization of social co-ordinations in differentiated societies: the theory of generalized symbolic media in Parsons, Luhmann and Habermas. The British Journal of Sociology, 53(3), 431-449. 
Coleman, J. S. (1964). Introduction to Mathematical Sociology. New York: Free Press of Glencoe.

Duong, D. V., \& Grefenstette, J. (2005). SISTER: a Symbolic Interactionist Simulation of Trade and Emergent Roles. Journal of Artificial Societies and Social Simulation, 8(1), 1. Retrieved from http://jasss.soc.surrey.ac.uk/8/1/1.html

Durkheim, E. (1982). Rules of Sociological Method. New York: Free Press.

Elder-Vass, D. (2005). Emergence and the realist account of cause. Journal of Critical Realism, 4(2), 315-338.

Elder-Vass, D. (2007a). For Emergence: Refining Archer's Account of Social Structure. Journal for the Theory of Social Behaviour, 37(1), 25-44.

Elder-Vass, D. (2007b). Luhmann and Emergentism: Competing Paradigms for Social Systems Theory? Philosophy of the Social Sciences, 37(4), 408-432.

Elster, J. (1989). Nuts and Bolts for the Social Sciences. New York: Cambridge University Press.

Emmeche, C., Køppe, S., \& Stjernfelt, F. (2000). Levels, Emergence, and Three Versions of Downward Causation. In P. B. Andersen, C. Emmeche, N. O. Finnemann, \& P. V. Christiansen (Eds.), Downward Causation. Minds, Bodies and Matter (pp. 13-34). Aarhus: Aarhus University Press.

Epstein, J. M. (1999). Agent-based computational models and generative social science. Complexity, 4(5), 41-60.

Epstein, J. M. (2007). Generative Social Science: Studies in Agent-Based Computational Modeling. New Jersey: Princeton University Press.

Epstein, J. M., \& Axtell, R. L. (1995). Growing Artificial Societies: Social Science from the Bottom Up. Washington, D.C.: Brookings Institution.

Fleischmann, A. (2005). A Model for a Simple Luhmann Economy. Journal of Artificial Societies and Social Simulation, 8(2), 4. Retrieved from http://jasss.soc.surrey.ac.uk/8/2/4.html

Fuchs, S. (1989). On the Microfoundations of Macrosociology: A Critique of Microsociological Reductionism. Sociological Perspectives, 32(2), 169-182.

Gilbert, N. (1995). Emergence in Social Simulation. In N. Gilbert \& R. Conte (Eds.), Artificial Societies: The Computer Simulation of Social Life (pp. 144-156). London: UCL Press.

Gilbert, N., \& Troitzsch, K. G. (2005). Simulation for the Social Scientist. Glasgow: Open University Press.

Gleick, J. (1988). Chaos: Making a New Science. London: Penguin.

Grant, W. E., Peterson, T. R., \& Peterson, M. J. (2002). Quantitative modeling of coupled natural/human systems: simulation of societal constraints on environmental action drawing on Luhmann's social theory. Ecological Modelling, 158(1-2), 143-165.

Hedström, P. (2005). Dissecting the Social: On the Principles of Analytical Sociology. Cambridge: Cambridge University Press.

Hedström, P., \& Bearman, P. (2011). The Oxford Handbook of Analytical Sociology. Oxford: Oxford University Press.

Hedström, P., \& Ylikoski, P. (2010). Causal Mechanisms in the Social Sciences. Annual Review of Sociology, 36(1), 49-67.

Hulswit, M. (2005). How Causal is Downward Causation? Journal for General Philosophy of Science, 36(2), 261-287. 
Kim, J. (2001). Making sense of downward causation. In P. B. Andersen, C. Emmeche, N. O. Finnemann, \& P. V. Christiansen (Eds.), Downward Causation: Minds, Bodies and Matter (pp. 305-321). Aarhus: Aarhus University Press.

Kron, T. (Ed.). (2002). Luhmann modelliert. Opladen: Verlag für Sozialwissenschaften.

Leydesdorff, L. (2005). Anticipatory Systems and the Processing of Meaning: a Simulation Study Inspired by Luhmann's Theory of Social Systems. Journal of Artificial Societies and Social Simulation, 8(2), 7. Retrieved from http://jasss.soc.surrey.ac.uk/8/2/7.html

Luhmann, N. (1988). Closure and Openness: On Reality in the World of Law. In G. Teubner (Ed.), Autopoietic law: a new approach to law and society (pp. 335-348). Berlin: Walter de Gruyter.

Luhmann, N. (1990). Essays on Self-Reference. New York: Columbia University Press.

Luhmann, N. (1994a). The Modernity of Science. New German Critique, (61, Special Issue on Niklas Luhmann), 9-23.

Luhmann, N. (1994b). Politicians, Honesty and the Higher Amorality of Politics. Theory, Culture and Society, 11(2), 25-36.

Luhmann, N. (1996). Social Systems. California: Stanford University Press.

Luhmann, N. (1999). Love as Passion: The Codification of Intimacy. California: Stanford University Press.

Luhmann, N. (2000). Art as a Social System. California: Stanford University Press.

Luhmann, N. (2004). Law as a Social System. Oxford: Oxford University Press.

Luhmann, N. (2007). La Sociedad de la Sociedad. México: Herder - Universidad Iberoamericana.

Manzo, G. (2007). Variables, Mechanisms, and Simulations: Can the Three Methods Be Synthesized? Revue française de sociologie, 48(5), 35-71.

Marchione, E., Salgado, M., \& Gilbert, N. (2010). “What did you say?” Emergent Communication in a Multi-Agent Spatial Configuration. Advances in Complex Systems, 13(04), 469-482.

Mascareño, A. (2008). Communication and Cognition: The Social Beyond Language, Interaction and Culture. Integrative Psychological and Behavioral Science, 42(2), 200-207.

Maturana, H., \& Varela, F. (1979). Autopoiesis and Cognition: The Realization of the Living. Kluwer Academic Publishers.

McLaughlin, B. (1992). The rise and fall of British emergentism. In A. Beckermann, H. Flohr, \& J. Kim (Eds.), Emergence or Reduction?: Essays on the Prospects of Nonreductive Physicalism. Berlin: Walter de Gruyter \& Co.

Parsons, T., \& Shils, E. (2001). Toward a General Theory of Action: Theoretical Foundations for the Social. New Jersey: Transaction Publishers.

Perfors, A. (2002). Simulated Evolution of Language: a Review of the Field. Journal of Artificial Societies and Social Simulation, 5(2), 4. Retrieved from http://jasss.soc.surrey.ac.uk/5/2/4.html

Sawyer, R. K. (2002). Emergence in Sociology: Contemporary Philosophy of Mind and Some Implications for Sociological Theory. American Journal of Sociology, 107(3), 551-585.

Sawyer, R. K. (2005). Social Emergence: Societies As Complex Systems. Cambridge: Cambridge University Press.

Schröder, J. (1998). Emergence: Non-Deducibility or Downwards Causation? The Philosophical Quarterly, 48(193), 433-452.

Squazzoni, F. (2008). The Micro-Macro Link in Social Simulation. Sociologica, (1/2008). 
Vanderstraeten, R. (2002). Parsons, Luhmann and the Theorem of Double Contingency. Journal of Classical Sociology, 2(1), 77-92.

Weber, M. (1978). Economy and Society: An Outline of Interpretive Sociology. California: University of California Press.

Winthrop-Young, G. (2003). On a Species of Origin: Luhmann’s Darwin. Configurations, 11(3), 305-349. 